CLAWAR 2019: 22nd International Conference on

Climbing and Walking Robots and the Support

Technologies for Mobile Machines,

Kuala Lumpur, Malaysia, 26-28 August 2019.

https://doi.org/10.13180/clawar.2019.26-28.08.21

\title{
CONCEPTUAL DESIGN OF 3D-PRINTED ACTIVE PROSTHETIC ARM WITH SOFT GRIPPERS FOR TODDLERS
}

\author{
DANIEL DE BARRIE and KHALED M. GOHER \\ School of Engineering, University of Lincoln, Brayford Way, Brayford Pool, \\ Lincoln, LN6 7TS, United Kingdom \\ E-mail:ddebarrie@lincoln.ac.uk,kgoher@lincoln.ac.uk \\ www.lincoln.ac.uk
}

\begin{abstract}
The present paper introduces a novel concept design for a 3D-printed prosthetic arm for toddlers, being developed to showcase the viability of early active prosthetic fitting that in due course could reduce prosthetic rejection rates. Soft grippers have been designed to act as the end device, with the intent to improve grasp capability of the prosthetic. The grippers have been incorporated into a prototype hand in order to display the functionally of the system. A CAD model of a stump has been visualised in Fusion 360 using its mesh feature, presenting an alternative to the traditional casting technique. An outline for the control system is also presented, using the position detector in a linear actuator to determine when a grasp has occurred. The concepts presented will further be worked on, with the realisation of a working prototype being the final intent.
\end{abstract}

\section{Introduction}

Congenital upper limb reduction defects occur in 4.1-5.0 per 10,000 births [1], with transverse deformities effecting around 2.9/10,000 [2]. Other non-congenital deformities will also effect infants, though there is limited work identifying the proportion effected; a 1988 to 1996 study found that dysvascular, trauma-related, and cancer related conditions had a respective frequency of $2.25,2.65$, and 0.15 per 100,000 between the ages of $0-14$ years [3]. Despite these figures, myoelectric prosthetics are customarily only given to adults, with the general consensus being that devices are unable to be scaled down to a paediatric level, in addition to being uneconomical due to the child's growth rate necessitating frequent replacements.

Congenital upper limb deficiencies, without the aid of a prosthetic, results in the patient recognising the stump as the end of the limb. This in turn during early childhood develops the motor neural skills and proprioception up to the base of the stump, resulting in the child developing their own methods of grasping objects [4], with later difficulty adapting to methods using a prosthetic device. Physical conditions as a result of limb loss are also present. Muscle atrophy occurs in cases where a lack of activity causes the muscle to weaken and reduces its size, this is apparent in amputees as the affected limb will normally be used less frequently and bare none or minimal load [5]. Targeted physiotherapy can reduce muscle atrophy, although this is not always readily available [6] and may be unworkable depending on the receptiveness of the child. The fitting of a prosthetic that allows functionally, such as grasping and lifting objects, will naturally develop these muscles, though some reduction in muscle mass will likely be present. This provides a muscular base for an eventual, larger adult prosthetic. 
In the long term, amputation may also cause issues with posture and muscular-skeletal pain [7] due to an overreliance on the residual limb and off balance centre of mass [8][9]. This in adulthood can cause deformation of the spine, affecting many aspects of life. The fitting of an active prosthetic can take the strain off the residual limb side and promotes a more natural, balanced approach to activities. Appropriate weighting of the prosthetic serves to realign the centre of mass, minimising asymmetrical posture and musculature [10].

Rejection of prosthetics proves to be a major challenge, however provided a level of comfort achieved and that the child can see the function of the device, the rejection rate is greatly reduced [11]. Early fitting too has been shown to reduce this risk [12], with one study showing a rejection rate for fitting before and after the age of 2 years of $22 \%$ and $58 \%$ respectively [13]. This work's proposed motorised prosthetic aims to counter prosthetic rejection based on this mantra of early adoption. The most recent study found that explores myoelectric infant prosthetics was conducted in 2002 [14]; a correlation between early adoption and duration of daily use was presented in this work. Issues of weight, reliability and battery life were highlighted by parents surveyed as part of the study, in line with the traditionally held notion of infant/child active prosthetics being too complex, expensive and heavy. This mantra is starting to shift with advancements in additive manufacturing based design and electrical components. This shift is highlighted by NHS England's policy to "cover future production of smaller child appropriate multi grip hands and digits" [15]. The proposed prosthetic aims to highlight this cost reduction and how early fitting could become more viable option, should the presented method be implemented.

In section 2, the design and manufacture of the soft grippers that will make up the end device of the prosthetic is looked at. Section 3 then examines the use of 3D printing within the context of prosthetics, including the use of 3D scanning techniques. Section 4 subsequently focuses on the control aspect of the proposed design, and finally conclusions and future work are presented.

\section{Soft Gripper Design and Manufacture}

\subsection{System Description}

The proposed design utilises cable driven soft grippers. A compressive selection process determined that this route, whilst currently unexplored, could provide the user with more capable end device than would be possible using a jointed hard material, like that currently used in most myoelectric prosthetics.

This style of the gripper has been investigated within the field of robotics [16], mostly in the picking of soft fruit [17] and other such activities that require a delicate grip, though the translation to prosthetics has not yet been made. This style utilises the elastic energy stored within a soft material to extend the gripper once the cables driving the flexion are relaxed, in this way we are able to negate the need for bidirectional drive of the grippers. 


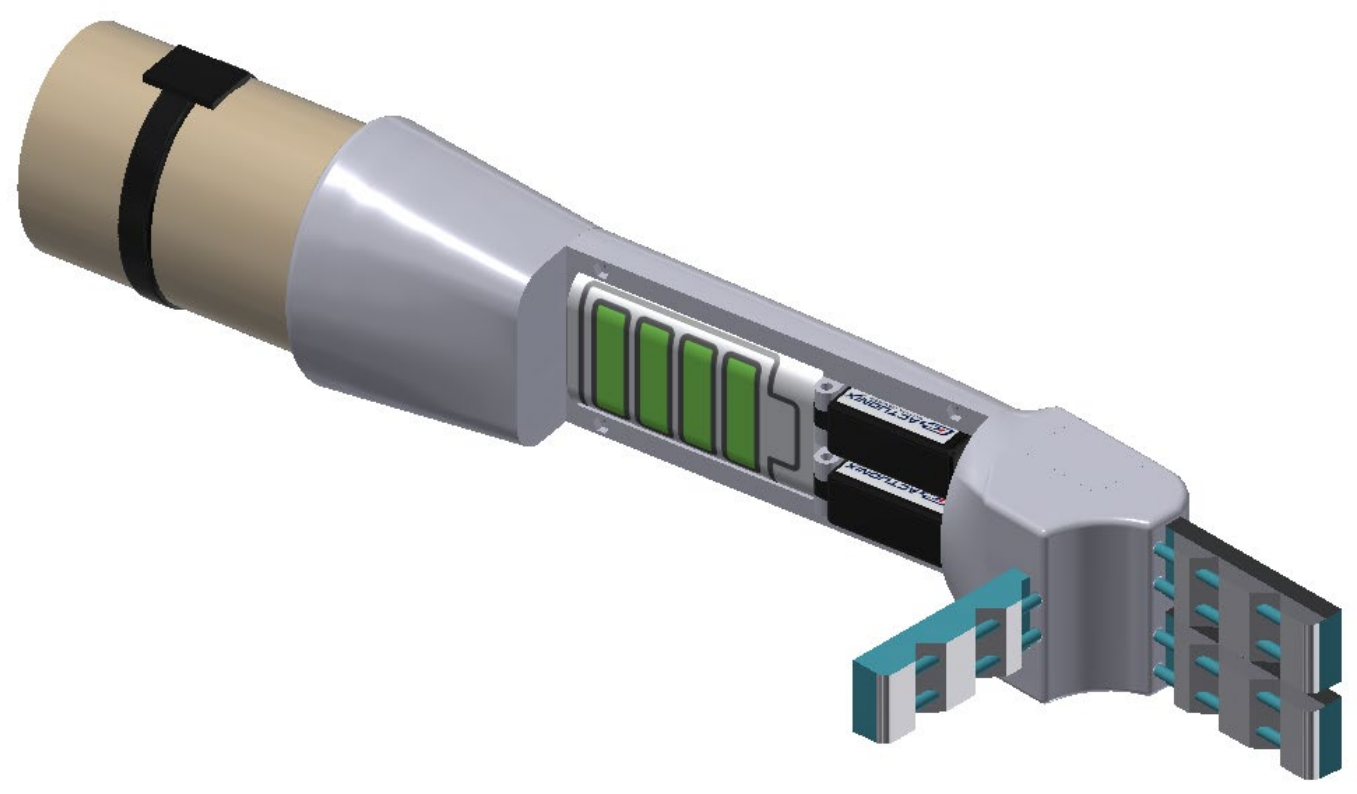

Figure 1. Concept Design for the Working Prototype Prosthetic Arm

\subsection{Design Details}

The design of the gripper is loosely based around the biological model of a human finger. The "fingers" are broken up into 3 phalanges, with $45^{\circ}$ triangular cuts separating the each segment. This provides a $90^{\circ}$ grip arch, with the proximal segment remaining mostly stationary, effectively acting as part of the palm. The proximal segment is also smaller than the medial and distal, as it plays less of a roll in active gripping. The grippers are made of two soft silicon materials; Smooth-Sil 960 (Smooth-On, Inc.) and Dragon Skin ${ }^{\circledR} 30$ (Smooth-On, Inc.). The Smooth-Sil 960 has a higher elastic modulus of approximately 5MPa, compared to $1 \mathrm{MPa}$ for the Dragon Skin 30. Both of these materials allow for the properties to be altered by adjusting the component mixing ratio. Based on these properties the Smooth-Sil 960 acts as the spline of the gripper, providing the elastic storage required for extension. Conversely the Dragon Skin 30 is often used prosthetic makeup, due to its skin like feel and subtleness. The combination of these two properties allows for a more balance grip performance. 


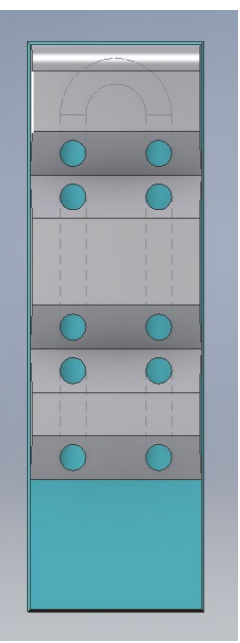

Figure 2. CAD model of gripper

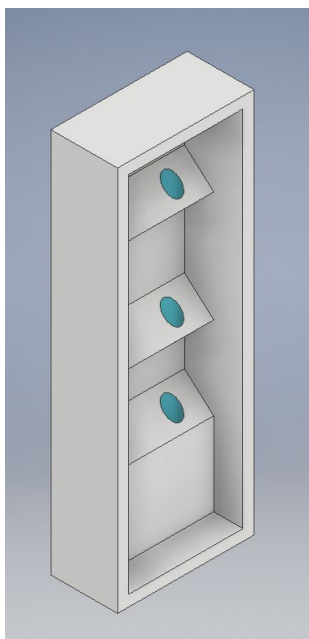

Figure 3. CAD model of gripper mould

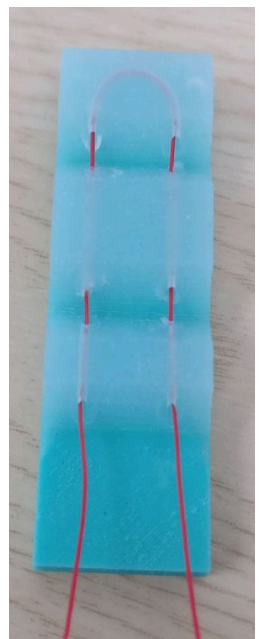

Figure 4. Final produced gripper with cable insert

\subsection{Manufacture}

The mould for the gripper, shown in fig.3, was created within Autodesk Inventor's mould environment, based off the gripper model in fig.2. The mould was 3D printed using TPU-95A, due to its flexibility aiding in part removal. $2 \mathrm{~mm}$ diameter plastic tubing segments are inserted into the mould. These form the channel for the cable to be fed into once the mould has set. The Dragon Skin 30 is added to the level of the triangular wedges and allowed to set, following this the Smooth-Sil 960 fills off the mould and is allowed to set. After mould removal, some small finishing techniques are required to remove excess material. A minimum finger width of $20 \mathrm{~mm}$ was determined due to the need for cable to be threaded through the loop in the distal segment. The final threaded gripper is shown in fig.4.

\section{3D Printing Technology and stump meshing}

\subsection{Overview of Additive Manufacturing Technologies for Prosthetics}

If done correctly 3D printing has the opportunity to drastically improve the design and production of prosthetic devices. The incorporation of 3D scanning provides an alternative to the traditional stump casting method, which is often uncomfortable, time consuming and in the case of young children sometimes traumatic. The current methods of manufacturing prosthetics have yet to incorporate these new technologies, though is it thought that adoption would be possible with relative ease [18].

\subsection{Hand/Arm Design}

The current prototype design utilises a three digit grip system, effectively 2 fingers and a thumb. J. Lenarčič et al [19] determined a grasp success rate of $90 \%$ can be achieved with a 3 digit system. The reduction in complexity and need for additional actuators is deemed to offset the visual disparity of a 3 vs 5 digit approach.

The hand was modelled on Autodesk Inventor and printed in ABS using the Ultimaker S5. The hand offsets the "fingers" and "thumb" by $90^{\circ}$, using an arched palm that allows for cylindrical grasp techniques, as well as pinch based methods.

This prototype requires the gripper cables to be pulled manually, this permitted initial testing of the designs grasp performance. The prototype was able to grasp a range of small 
objects, using different grasping techniques as shown in fig. 5 - 7. Further, more thorough validation of the grasp performance will be performed in due course, along with revisions to the design that will eventually be incorporated into the prosthetic arm.

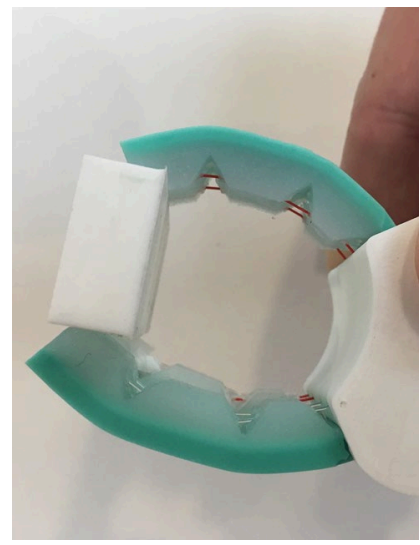

Figure 5. Pinch grip of a rubber block

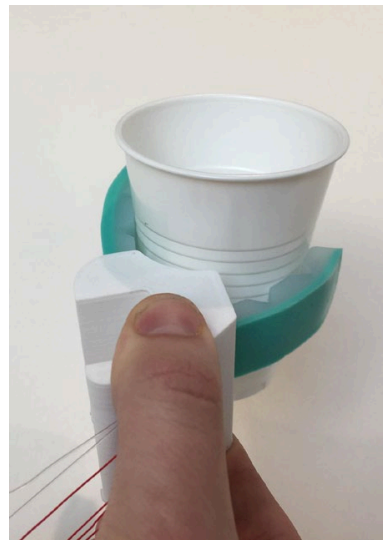

Figure 6. Cylindrical grip of a plastic cup

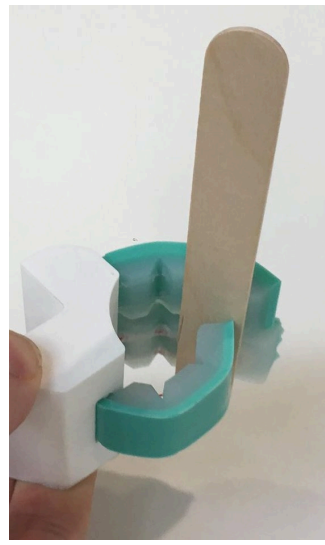

Figure 7. Pinch grip of a wooden stick

\subsection{Stump Meshing}

CAD systems such as Autodesk Fusion 360 utilise a mesh tool that allows for the modelling of geometrically complex shapes by segmenting the model's surface into many tiles that can be individually manipulated in a very tactile way. This system of modelling lends itself to the process of socket design, where the stump geometry would make dimensional based CAD modelling very difficult.

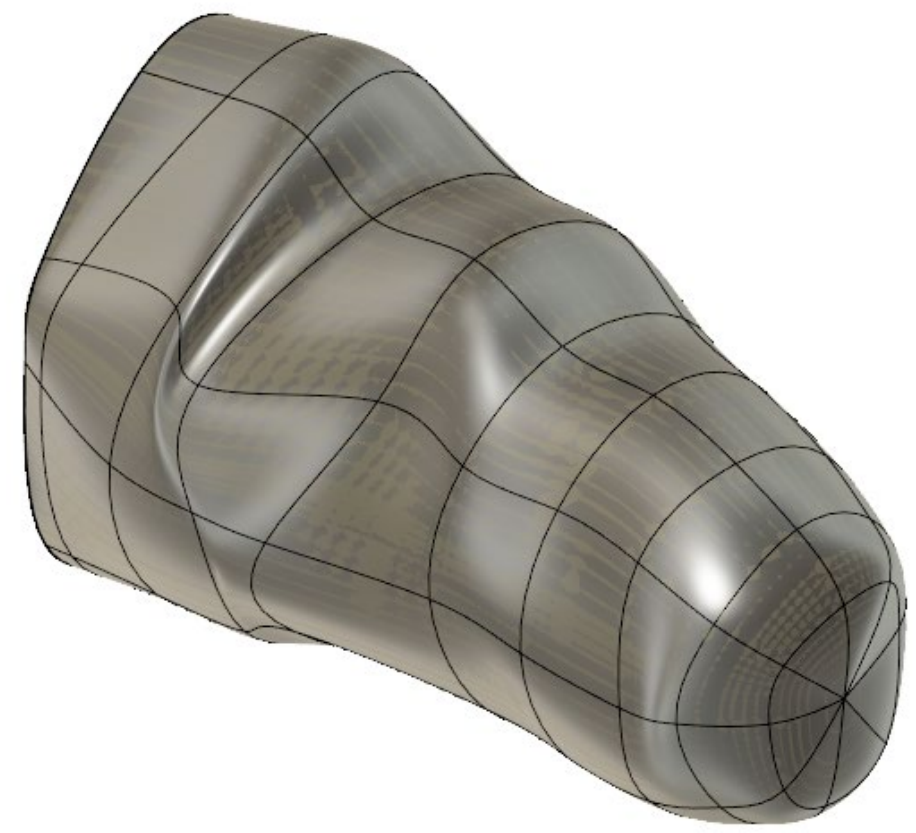

Figure 8. CAD model of an example stump using the mesh technique 
As a proof of concept, an example scan of a stump has been meshed in Fusion 360, as shown in fig.8. Much like with casting based methods, this socket would vary between individuals and is greatly reliant on the skill of the prosthetists. One major advantage of using this method is the speed at with the socket can be designed and produced, currently the process takes months, often resulting in the child outgrowing the socket, prior to fitting.

\section{Control System Design}

The proposed control method is a single point, single action voluntary opening myoelectric system. The method involves the hand being in a closed position by default, with the hand voluntarily opening with a detected muscle flex. Initially the sensitivity of the system can be set quite high, so that the child becomes aware of the hand's movement. As they begin to master the gesture, the sensitivity can be reduced. This system is amongst the most simple for a myoelectric prosthetic, with the purpose being to introduce the concept to children at a young age, preparing them for a subsequent more complex multi-site, multiple gesture system. The electromyography (EMG) sensor placement is of key importance. Current designs aim to use an armband based sensor, such as the OYMotion Gravity controller. This would allow sensor to be placed on the upper arm, where the length of the residual limb would not affect sensor placement as would be the case if the sensor were to be integrated into the socket itself.

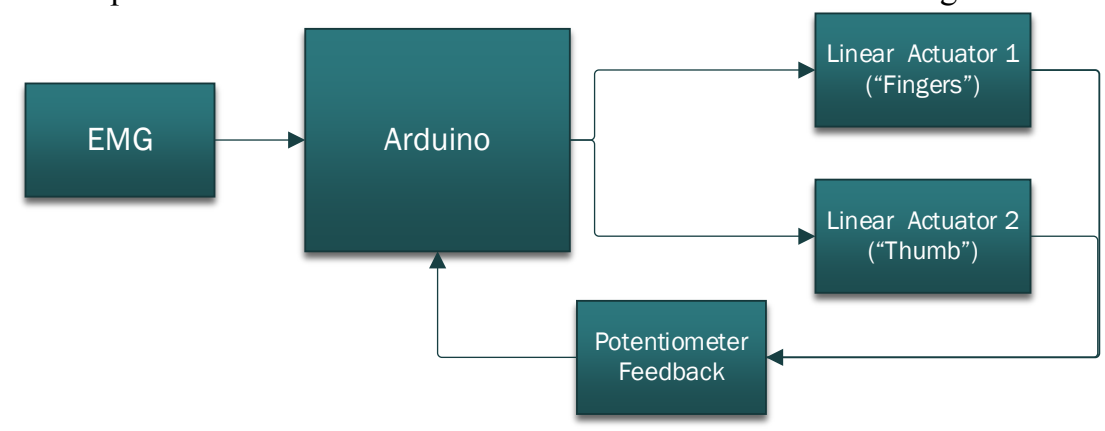

Figure 9. Proposed control system outline

The completed prosthetic will use 2 Actuonix PQ12-P Micro Linear Actuators to drive the grippers. This small scale linear actuator contains within it a potentiometer providing analogue feedback of the shaft position. Limits for fully open and closed positions can be set using this feedback. A method for grasp detection is also proposed, this will utilise the position feedback to determine the error between the expected and detected movement of the actuator. Should the error exceed the set threshold, then this is indicative of a stall i.e. contact with an object. Fig.9 displays an outline of the relationship between the primary components within the control system. This system will be developed in Simulink using the Arduino toolbox for eventual incorporation into a working prototype prosthetic.

\section{Conclusion}

Aiming to showcase the viability of a myoelectric upper-limb prosthetic for toddlers, this paper presents the concept design for a novel 3D-printed device, utilising soft grippers. Prosthetic rejection remains a major issue, with many potential long term health consequences for amputees. It is hoped that such a design will allow the concept of young prosthetic fitting to be re-evaluated, in line with a growing body of work highlighting benefits of early adoption in minimising rejection rates. The use of $3 \mathrm{D}$ printing allows for the rapid manufacture of 
custom devices, in doing so reducing cost of such a device, opening up the possibility of active prosthetics being financially viable for healthcare providers to supply them to young children. In addition, soft grippers look to improve on the traditional jointed fingers normally used in prosthetics. The elimination of these fixed joints allows for a more adaptive grip, as well as removing a level of complexity that has hindered the design of small scale prosthetic hands/gripping devices. A simple voluntary opening control system is proposed with the intention of being a child's introduction to myoelectric control, setting a precedent for future prosthetics. Future considerations of this work will focus on integrating the concepts presented here into a working prototype of a complete prosthetic limb and evaluate it against activities of daily living (ADLs).

\section{References}

[1] E. Vasluian et al., "Birth prevalence for congenital limb defects in the northern Netherlands: A 30-year population-based study," BMC Musculoskelet. Disord., vol. 14, 2013.

[2] T. Bedard, R. B. Lowry, B. Sibbald, G. N. Kiefer, and A. Metcalfe, "Congenital limb deficiencies in Alberta-A review of 33 years (1980-2012) from the Alberta Congenital Anomalies Surveillance System (ACASS)," Am. J. Med. Genet. Part A, vol. 167, no. 11, pp. 2599-2609, 2015.

[3] T. R. DILLINGHAM, L. E. PEZZIN, and E. J. MACKENZIE, "Limb Amputation and Limb Deficiency: Epidemiology and Recent Trends in the United States," South. Med. J., vol. 95, no. 8, pp. 875-883, 2002.

[4] H. Mano, S. Fujiwara, and N. Haga, "Adaptive behaviour and motor skills in children with upper limb deficiency," Prosthet. Orthot. Int., vol. 42, no. 2, pp. 236-240, 2018.

[5] T. Schmalz, S. Blumentritt, and C. D. Reimers, "Selective thigh muscle atrophy in trans-tibial amputees: An ultrasonographic study," Arch. Orthop. Trauma Surg., vol. 121, no. 6, pp. 307-312, 2001.

[6] J. Treby and E. Main, "A survey of physiotherapists involved in paediatric lower limb amputee rehabilitation in the British Isles," Physiotherapy, vol. 93, no. 3, pp. 212-217, 2007.

[7] M. Allami et al., "A comprehensive musculoskeletal and peripheral nervous system assessment of warrelated bilateral upper extremity amputees," Mil. Med. Res., vol. 3, no. 1, pp. 1-8, 2016.

[8] S. Postema, Upper limb absence: Effects on body functions and structures, musculoskeletal complaints, and functional capacity. 2017.

[9] H. J. Rinne, "Late Sequelae of Amputation, a Summary," vol. 54, 1965.

[10] B. Greitemann, V. Güth, and R. Baumgartner, "Asymmetry of posture and trunk musculature following unilateral upper limb amputation: A clinical, electromyographic, posture-analytical and photogrammetric study [Asymmetrie der Haltung und der Rumpfmuskulatur nach einseitiger Armamputation - Eine klinis," Z. Orthop. Ihre Grenzgeb., vol. 134, no. 6, pp. 498-510, 1996.

[11] K. Postema, V. Van Der Donk, J. Van Limbeek, R. A. J. Rijken, and M. J. Poelma, "Prosthesis rejection in children with a unilateral congenital arm defect," Clin. Rehabil., vol. 13, no. 3, pp. 243-249, 1999.

[12] M. Meurs, C. G. B. Maathuis, C. Lucas, M. Hadders-Algra, and C. K. van der Sluis, "Prescription of the first prosthesis and later use in children with congenital unilateral upper limb deficiency: A systematic review," Prosthet. Orthot. Int., vol. 30, no. 2, pp. 165-173, 2006.

[13] T. Scotland and H. Galway, "A long-term review of children with congenital and acquired upper limb deficiency,” J. Bone Joint Surg. Br., vol. 65-B, no. 3, pp. 346-349, 1983.

[14] M. Egermann, P. Kasten, and M. Thomsen, "Myoelectric hand prostheses in very young children," Int. Orthop., vol. 33, no. 4, pp. 1101-1105, 2009.

[15] NHS England, "Clinical Commissioning Policy: Multi-grip Upper Limb Prosthetics," pp. 1-26.

[16] M. Manti, T. Hassan, G. Passetti, N. D’Elia, C. Laschi, and M. Cianchetti, "A Bioinspired Soft Robotic Gripper for Adaptable and Effective Grasping," Soft Robot., vol. 2, no. 3, pp. 107-116, 2015.

[17] V. Slesarenko, S. Engelkemier, P. I. Galich, D. Vladimirsky, G. Klein, and S. Rudykh, "Strategies to control performance of 3D-printed, cable-driven soft polymer actuators: From simple architectures to gripper prototype," Polymers (Basel)., vol. 10, no. 8, 2018.

[18] S. J. Day and S. P. Riley, "Utilising three-dimensional printing techniques when providing unique assistive devices: A case report," Prosthet. Orthot. Int., vol. 42, no. 1, pp. 45-49, 2018.

[19] J. Lenarčič, T. Bajd, and M. M. Stanišić, "Robot Grasp,” 2013, pp. 291-311. 